\title{
Inverting Laguerre Tessellations
}

\author{
Qibin Duan ${ }^{1}$, Dirk P. Kroese ${ }^{1}$, Tim Brereton ${ }^{2}$, Aaron Spettl $^{2}$ \\ AND VOLKER SCHMIDT ${ }^{2}$ \\ ${ }^{1}$ School of Mathematics and Physics, The University of Queensland, Brisbane 4072, Australia \\ ${ }^{2}$ Institute of Stochastics, Ulm University 89069 Ulm, Germany \\ Email: qbduan@gmail.com; kroese@maths.uq.edu.au
}

\begin{abstract}
A Laguerre tessellation is a generalization of a Voronoi tessellation where the proximity between points is measured via a power distance rather than the Euclidean distance. Laguerre tessellations have found significant applications in materials science, providing improved modeling of (poly)crystalline microstructures and grain growth. There exist efficient algorithms to construct Laguerre tessellations from given sets of weighted generator points, similar to methods used for Voronoi tessellations. The purpose of this paper is to provide theory and methodology for the inverse construction; that is, to recover the weighted generator points from a given Laguerre tessellation. We show that, unlike the Voronoi case, the inverse problem is in general non-unique: different weighted generator points can create the same tessellation. To recover pertinent generator points we formulate the inversion problem as a multimodal optimization problem and apply the cross-entropy method to solve it.
\end{abstract}

Keywords: Laguerre tessellation, power diagram, power distance, inverse problem, generator points, multimodal optimization, cross-entropy method

Received 00 November 2013

\section{INTRODUCTION}

The Voronoi tessellation of a given set of generator points in the Euclidean plane divides the plane into disjoint cells (regions, tiles), such that, for all points in a cell, the Euclidean distance to the generator point within that cell is less than the distance to all other generator points. Applications of Voronoi tessellations may be found in fields as diverse as computational geometry, cell-biology, architecture, image analysis, ecology, and materials science. Various generalizations of the Voronoi tessellation have been proposed and investigated to better fit tessellations appearing in nature. These include tessellations that use different distance metrics and tessellations that use different geometric objects (e.g., lines and areas, instead of points); see, for example, [1].

An important generalization is the Laguerre tessellation, also called power diagram, which employs weighted generator points and uses the power distance to measure the proximity of points; see, e.g., $[1,2,3]$. It has been shown that many convex tilings in three or more dimensions are Laguerre tessellations (see [3]), and also in two dimensions Laguerre tessellations are common. In addition, Voronoi tessellations in a number of nonEuclidean geometries can be represented as Laguerre tessellations (see [4]). For these reasons, Laguerre tessellations have been a popular choice for modeling grain growth structures (see $[5,6]$ ) and boundaries of poly- crystalline materials (see [7]).

In the statistical analysis of spatial data described by Laguerre tessellations (for example, in the study of microscopic materials and cell-tissue), it is often the case that the positions of the generator points are unknown. Knowledge of these sites is useful for statistical inference on the properties of random tessellations. The problem of inverting Voronoi tessellations - that is, recovering the generator points from the tessellation - has been well studied; see, for example, $[8,9,10,11,12,13]$. However, the inversion problem for Laguerre tessellations seems to be much less studied. Recently, an approximate inversion method was given in [14], although with a different motivation, i.e., the possibility of reconstructing the complete tessellation using only cells' centers of mass and cell volumes. An alternative approach was presented in [15], where experimental 3D data was described by extraction of (parametric) cells using orthogonal regression - but without using Laguerre tessellations.

The goal of the current paper is to provide a better understanding of the inversion properties of Laguerre tessellations, and to formulate a simple and effective method for computing such inversions for 2D Laguerre tessellations. We find that, in contrast to Voronoi tessellations, the Laguerre inversion problem admits many solutions. We identify pertinent solutions with useful optimality properties. Our method involves a minimax optimization problem that is solved via the 
cross-entropy method ([16]).

The rest of this paper is organized as follows. In Section 2 we provide the mathematical background for Laguerre tessellations. In Section 3 we give the problem description and show that there may be multiple solutions to the inversion problem. We provide a simple algorithm to generate possible solutions, and discuss a minimization approach to obtain pertinent generator points. Section 4 details the cross-entropy algorithm for finding generator points that minimize the maximum weight (radius) of the Laguerre generator points. Numerical experiments in Section 5 demonstrate the effectiveness of the approach. Finally, Section 6 gives the conclusions and proposes directions for future research.

\section{LAGUERRE TESSELLATIONS}

A Laguerre tessellation, also called a power diagram or a Laguerre diagram, is a weighted version of the wellknown Voronoi tessellation. In this section we introduce the mathematical notation and review some basic facts about these objects. See [1] and [2] for more details on Laguerre tessellations.

\subsection{Definitions}

Let $p \in \mathbb{R}^{d}$ be a fixed point and $w \in \mathbb{R}$ be a fixed value, called the weight of point $p$. We call the pair $(p, w)$ a weighted point. For all $x \in \mathbb{R}^{d}$, we define the power of $x$ with respect to $(p, w)$ as

$$
\operatorname{pow}(x,(p, w))=\|x-p\|^{2}-w .
$$

Suppose $\mathbf{P}=\left\{\left(p_{1}, w_{1}\right),\left(p_{2}, w_{1}\right), \ldots,\left(p_{n}, w_{n}\right)\right\}$ is a (finite) set of weighted (generator) points in $\mathbb{R}^{d}$. The Laguerre tessellation of $\mathbf{P}$ divides $\mathbb{R}^{d}$ into cells, using the power of these points. The cell associated with the $i$-th generator point, $C_{i}$, is defined by

$C_{i}=\left\{x \in \mathbb{R}^{d}: \operatorname{pow}\left(x,\left(p_{i}, w_{i}\right)\right) \leqslant \operatorname{pow}\left(x,\left(p_{j}, w_{j}\right)\right), i \neq j\right\}$.

Note that if all the weights are equal, the Laguerre tessellation reduces to the standard Voronoi tessellation.

REMARK 1. Laguerre tessellations on a locally finite but possibly infinite set of generator points are defined in the same way.

When all weights are positive, each weighted generator point $(p, w) \in \mathbf{P}$ can be interpreted and visualized as a sphere (denoted by $S(p, r)$ ) with radius $r=\sqrt{w} \geqslant 0$ centered at point $p$. The power of a point $x$ with respect to the sphere $S(p, r)$ is thus given by

$$
\operatorname{pow}(x, S(p, r))=\|x-p\|^{2}-r^{2} .
$$

Geometrically, this means that for a point $x$ outside the sphere $S(p, r)$, the value of $\operatorname{pow}(x, S(p, r))$ is equal to the squared length of the tangent line from $x$ to $S(p, r)$.
TABLE 1. Tessellation storage format

\begin{tabular}{cccccc} 
cell $i$ & cell $j$ & \multicolumn{4}{c}{ edge $e_{i, j}$} \\
& & $v_{1}(x)$ & $v_{1}(y)$ & $v_{2}(x)$ & $v_{2}(y)$ \\
\hline 1 & 4 & 126.14 & 138.02 & 140.86 & 156.07 \\
1 & 11 & 98.75 & 150.55 & 126.14 & 138.02 \\
1 & 14 & 93.26 & 164.39 & 98.75 & 150.55 \\
2 & 6 & 100.97 & 84.85 & 107.45 & 89.69 \\
2 & 10 & 107.45 & 89.69 & 112.99 & 113.46 \\
2 & 11 & 112.99 & 113.45 & 83.46 & 126.78
\end{tabular}

The boundary between two adjacent cells generated by spheres $S_{1}=S\left(p_{1}, r_{1}\right)$ and $S_{2}=S\left(p_{2}, r_{2}\right)$, consists of all points $z \in \mathbb{R}^{d}$ such that $\operatorname{pow}\left(z, S_{1}\right)=\operatorname{pow}\left(z, S_{2}\right)$. These points form a hyperplane $H\left(S_{1}, S_{2}\right)$, where

$H\left(S_{1}, S_{2}\right)=\left\{z \in \mathbb{R}^{d}: 2\left\langle z, p_{1}-p_{2}\right\rangle=\left\|p_{1}\right\|^{2}-\left\|p_{2}\right\|^{2}+r_{2}^{2}-r_{1}^{2}\right\}$.

This boundary is perpendicular to the line joining $p_{1}$ and $p_{2}$ and is called the radical axis of $S_{1}$ and $S_{2}$.

In this paper, for simplicity, we will only consider Laguerre tessellations in $\mathbb{R}^{2}$. In this case, the generators can be interpreted as circles.

\subsection{Representations}

A Laguerre tessellation can be represented mathematically as a geometric graph - a collection of vertices $\mathbf{V}=\left\{v_{1}, \ldots, v_{m}\right\}$ (also $\left.\mathbf{V}(\mathbf{P})\right)$ and edges $\left\{\left(v_{i}, v_{j}\right)\right\}$, where the vertices are assigned positions in space. Note that some cells are not only bounded by segments but also by rays that extend to infinity in a certain direction, because we consider finite sets of generators. Such edges are often represented using a 'dummy' vertex. That is, one vertex of the edge is set to be an arbitrary point on the ray extending to infinity, see [12]. Interior vertices of the tessellation have equal power with respect to at least three separate circles. In contrast, 'dummy' vertices only have equal power with respect to two circles.

Using the above representation, the vertices and edges of the Laguerre tessellations can be stored in the format given in Table 1. The cells are labeled from 1 to $n$. The first two columns of Table 1 correspond to the labels of adjacent cells. For example, cell 1 is adjacent to cells 4,11 , and 14 . The coordinates of the vertices of the edge that separates two cells are given in columns 3-6.

A normal tessellation is one in which adjacent cells are face-to-face, i.e., (in 2D) they share edges and vertices; furthermore, each edge borders exactly two cells, and each vertex is shared by exactly three cells. An example of a normal 2D Laguerre tessellation, generated by 15 circles, is given in Figure 1. The boundaries between the cells are the edges of the geometric graph. The powers of the points in each boundary are equal with respect to the two neighboring circles. Notice that the degree of each (interior) vertex 
is 3 . The powers of the vertices are therefore equal with respect to three neighboring circles.

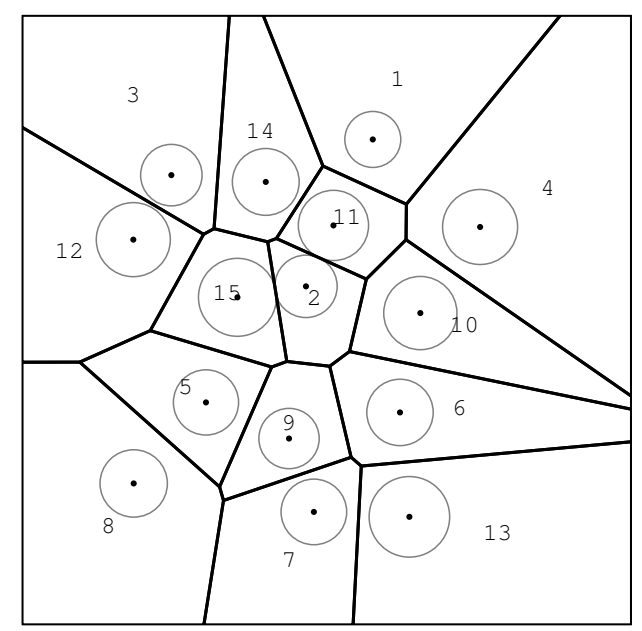

FIGURE 1. Laguerre tessellation for 15 circles

Typical 2D Laguerre tessellations are normal. For example, when the generator points are randomly and uniformly chosen within a bounded sampling window, the tessellation is normal with probability 1. From now on, we consider only normal tessellations.

\subsection{Properties}

We next discuss some properties of Laguerre tessellations which are important for the problem of inverting Laguerre tessellations.

Property 1. A Laguerre cell does not necessarily contain its generator and a generator does not necessarily generate a cell.

Figure 2 (from $[2,3]$ ), shows that the generator point of a Laguerre cell can lie outside its cell; in particular, $p_{4}$ lies outside cell 4 . The same figure shows a generator circle, $S\left(p_{6}, r_{6}\right)$, for which the corresponding Laguerre cell is empty.

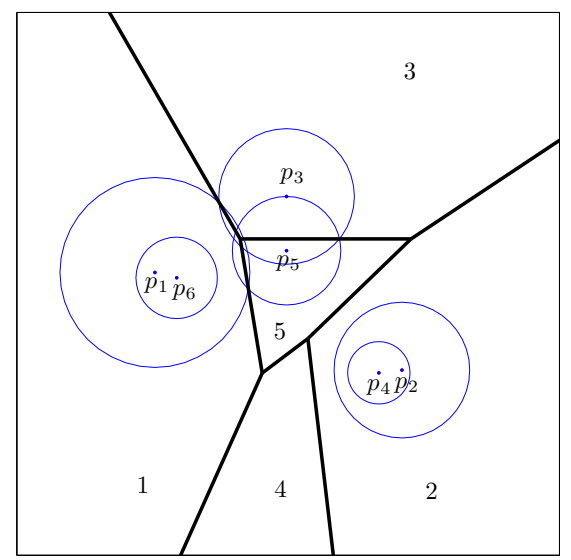

FIGURE 2. Laguerre tessellation for six circles
A consequence of Property 1 is that the generator set for a given tessellation is not unique. Namely, one can add circles that do not generate additional cells, and the new set will give the same Laguerre tessellation as the original one.

However, even when each circle generates a cell, the generators of a Laguerre tessellation are not necessarily unique.

Property 2. Two completely different sets of circles can generate the same Laguerre tessellation.

An extreme example is given in Figure 3, where both the gray circles and yellow circles yield the same tessellation. The method for constructing such generating circles is discussed in Section 3.

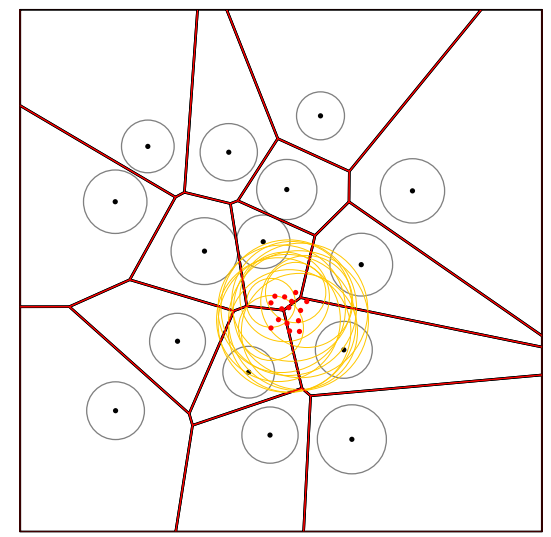

FIGURE 3. One Laguerre tessellation generated by two completely different sets of circles

\section{PROBLEM DESCRIPTION}

The problem of efficiently generating tessellations has been extensively studied in computer science and computational geometry. In this problem, the generators of the tessellation are given, but the tessellation itself is unknown. A less studied problem, but one with extensive applications, is the inverse problem. In this problem, the tessellation is given, but the generators are unknown.

In the case of Voronoi tessellations, the inverse problem has a number of elegant solutions. See, for example, [13, 12, 8, 10, 11]. Unfortunately, these approaches do not easily extend to the Laguerre case. One reason for this is that the inverse Voronoi problem has more structure than the inverse Laguerre problem. Another reason is that the inverse Voronoi problem has a unique solution, whereas the inverse Laguerre problem in general has an infinite number of solutions.

Finding a set of weighted generating points that generate a given Laguerre tessellation is not too difficult; see Algorithm 1 below. However, finding pertinent solutions - that is, more suitable, meaningful solutions - is considerably harder. Pertinent solutions possess extra structure that is imposed by the modeler. Typical requirements arising in materials science (see, 
e.g., [14]), geometry (e.g., sphere packing [17]), molecular biology and biochemistry (see, e.g., [18]), include the following:

1. The weights of the generating points should be non-negative, so that the weighted points can be interpreted as circles.

2. The generating points should ideally lie within the cells they generate and, if they do not, they should not be too far away.

3. The maximum radius should be as small as possible.

4. The average radius should correspond to a circle whose average volume equals the average volume of a cell.

5. The generating points should be close to the centers of mass of the cells.

\subsection{Weighted points that generate a given Laguerre tessellation}

We begin by describing how, for a given (normal) tessellation, a set of weighted generator points can be determined by specifying only the coordinates and weight of one weighted generator point and one coordinate of the weighted generator point of a neighboring cell.

THEOREM 3.1. The weighted generator points of a given normal 2D Laguerre tessellation can be entirely determined from the weighted generator point of one interior cell, and one coordinate of the weighted generator point of an adjacent cell.

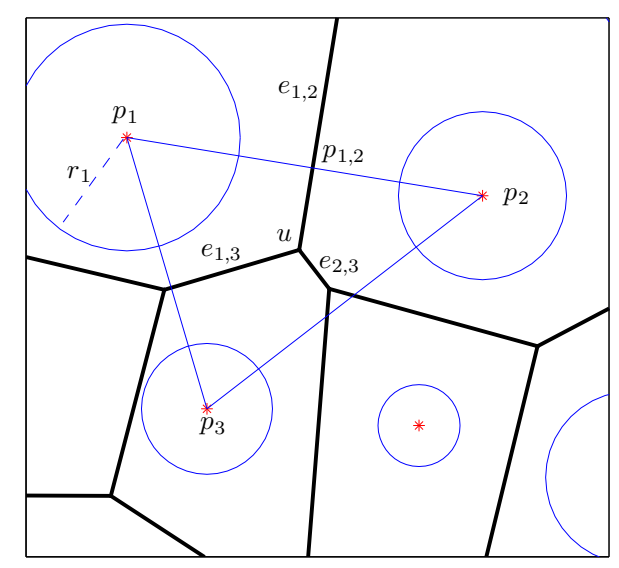

FIGURE 4. Two generators determine the third

Proof. For simplicity we assume that the weights of the generator points are positive, although the proof does not use this assumption - the advantage is that the weighted generator points can be interpreted as circles; see Figure 4. Let $S_{1}\left(p_{1}, r_{1}\right)$ be the generator circle of some interior cell, $C_{1}$, and let $S_{2}\left(p_{2}, r_{2}\right)$ be the generator circle of an adjacent cell, $C_{2}$. Let the coordinates of $p_{1}$ and $p_{2}$ be $\left(x_{1}, y_{1}\right)$ and $\left(x_{2}, y_{2}\right)$, respectively. The assumption of the theorem is that $x_{1}, y_{1}, r_{1}$, and $x_{2}$ are given.

Let $e_{1,2}$ be the edge of the tessellation separating $C_{1}$ and $C_{2}$. For Laguerre tessellations, the line segment connecting $p_{1}$ and $p_{2}$ is perpendicular to $e_{1,2}$. Let $m_{1,2}$ be the slope of $e_{1,2}$. It follows that $y_{2}$ is determined by

$$
\frac{y_{2}-y_{1}}{x_{2}-x_{1}}=-\frac{1}{m_{1,2}} \text {. }
$$

(Note that while it is possible that $m_{1,2}=0$, this is not relevant in practical applications. The problem can be solved, for example, by rotating the tessellation. Therefore, we assume the slope to be non-zero.) From this we can determine $r_{2}$ via

$$
\left\|p_{1}-q\right\|^{2}-r_{1}^{2}=\left\|p_{2}-q\right\|^{2}-r_{2}^{2}
$$

for any point $q$ on the line containing segment $e_{1,2}$. In particular, we can take $q=p_{1,2}$, the intersection of the line through $p_{1}$ and $p_{2}$, and the line containing the edge $e_{1,2}$.

Because the tessellation is assumed to be normal and $C_{1}$ is an interior cell, there is a cell adjacent to both $C_{1}$ and $C_{2}$, say $C_{3}$. The generating circle of $C_{3}$, $S_{3}=\left(p_{3}, r_{3}\right)$, is determined as follows. The point $p_{3}=\left(x_{3}, y_{3}\right)$ is the intersection of (1) the line that passes through $p_{1}$ and is perpendicular to $e_{1,3}$ (the edge between $C_{1}$ and $C_{3}$ ) and (2) the line that passes through $p_{2}$ and is perpendicular to $e_{2,3}$ (the edge between $C_{2}$ and $C_{3}$ ). It follows that the coordinates $x_{3}$ and $y_{3}$ of $p_{3}$ satisfy

$$
\frac{y_{3}-y_{1}}{x_{3}-x_{1}}=-\frac{1}{m_{1,3}} \quad \text { and } \quad \frac{y_{3}-y_{2}}{x_{3}-x_{2}}=-\frac{1}{m_{2,3}},
$$

where $m_{1,2}$ and $m_{2,3}$ are the slopes of $e_{1,2}$ and $e_{2,3}$, respectively. Hence,

$$
\begin{aligned}
x_{3} & =\frac{m_{2,3}\left(m_{1,3} y_{1}+x_{1}\right)-m_{1,3}\left(m_{2,3} y_{2}+x_{2}\right)}{m_{2,3}-m_{1,3}} \\
y_{3} & =\frac{m_{1,3} y_{1}+x_{1}-\left(m_{2,3} y_{2}+x_{2}\right)}{m_{1,3}-m_{2,3}} .
\end{aligned}
$$

The radius, $r_{3}$, is then determined as in (2); that is,

$$
\left\|p_{1}-q\right\|^{2}-r_{1}^{2}=\left\|p_{3}-q\right\|^{2}-r_{3}^{2}
$$

where $q$ is any point on the line containing the edge $e_{1,3}$.

It is also possible to determine $r_{3}$ by considering the pair $C_{2}, C_{3}$ rather than $C_{1}, C_{3}$, giving

$$
\left\|p_{2}-q\right\|^{2}-r_{2}^{2}=\left\|p_{3}-q\right\|^{2}-r_{3}^{2}
$$

where $q$ is any point on the line containing the edge $e_{2,3}$. To prove that (5) and (6) give the same value for $r_{3}^{2}$, it suffices to show that

$$
r_{1}^{2}-\left\|p_{1}-u\right\|^{2}=r_{2}^{2}-\left\|p_{2}-u\right\|^{2},
$$


where $u$ is the vertex on the intersection of the lines containing edges $e_{1,2}$ and $e_{2,3}$. But this follows directly from the definition of the Laguerre tessellation, and the fact that $u$ lies also on the line through edge $e_{1,2}$.

Proceeding in this fashion, it is possible to iteratively determine the generator circle of each cell.

REMARK 2. Theorem 3.1 was given in $2 \mathrm{D}$, but the idea of the proof is applicable for higher dimensions. Using normal vectors for hyperplanes separating cells, e.g., edges in $2 \mathrm{D}$ or planar faces in $3 \mathrm{D}$, it is clear that generators have to lie on lines with the same orientation. See also [19], where a related non-unique construction is given for the orthogonal dual of a Laguerre tessellation (in the Voronoi case this corresponds to the Delaunay triangulation).

REMARK 3. The proof of Theorem 3.1 shows that if we add the same constant to all squared radii, the tessellation does not change. Namely, if $\left\|p_{i}-q\right\|^{2}-r_{i}^{2}=$ $\left\|p_{j}-q\right\|^{2}-r_{j}^{2}$ for every $q$ on an edge separating adjacent cells $C_{i}$ and $C_{j}$, then the same is true if $r_{i}^{2}$ and $r_{j}^{2}$ are replaced by $r_{i}^{2}+c$ and $r_{j}^{2}+c$. As a consequence, it is always possible to find a set of generators that all have positive weights. If some weights are negative, we simply find the minimum of these and subtract this value from all weights.

Theorem 3.1 and Remark 3 suggest the following algorithm for determining the generator circles of a Laguerre tessellation, given $x_{1}, y_{1}, r_{1}$ and $x_{2}$. Note that in the algorithm we start with a pair of (internal) cells $\left(C_{1}, C_{2}\right)$. This may be replaced with any pair of internal cells by relabeling.

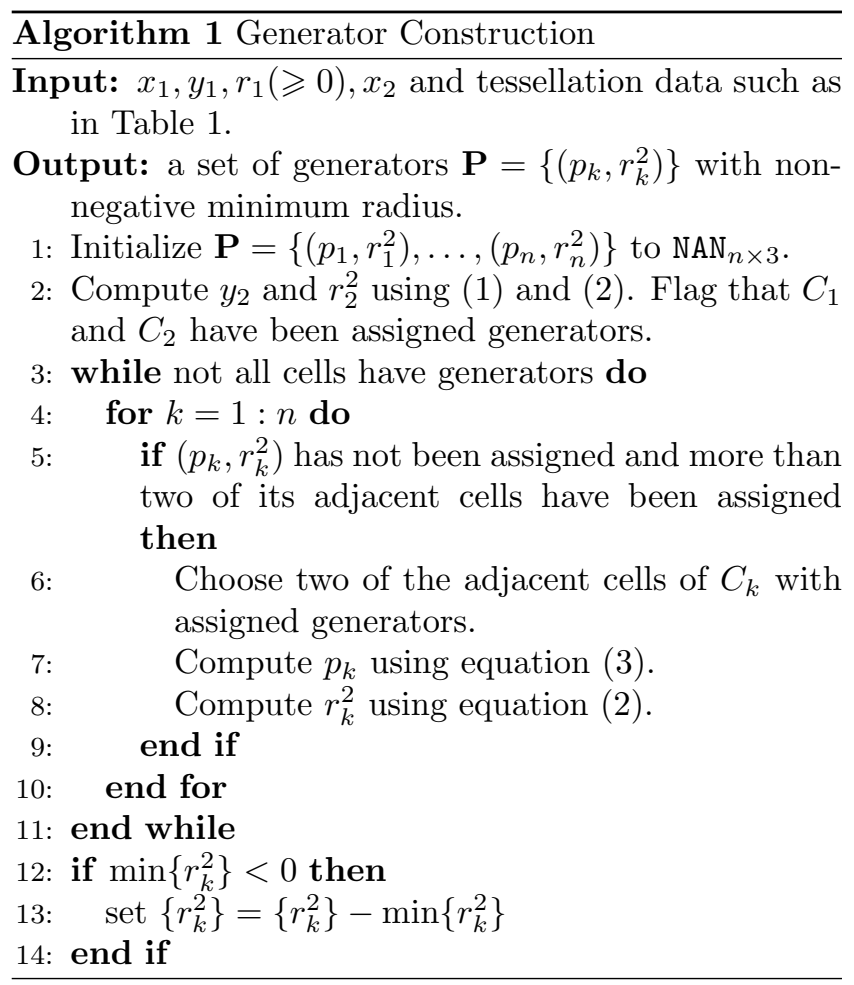

\subsection{Choosing a pertinent solution}

Algorithm 1 provides a method for recovering the generating circles of a tessellation given a small number of inputs, namely $x_{1}, y_{1}, r_{1}$, and $x_{2}$. However, depending on how these inputs are chosen, very different results can be obtained. Although the algorithm guarantees positive weights, in some cases the generating circles can lie far outside the cells they generate. An example is given in Figure 3. In other cases, the radii of the generating circle can be much bigger than the cells or even the observation window; see Figure 5.

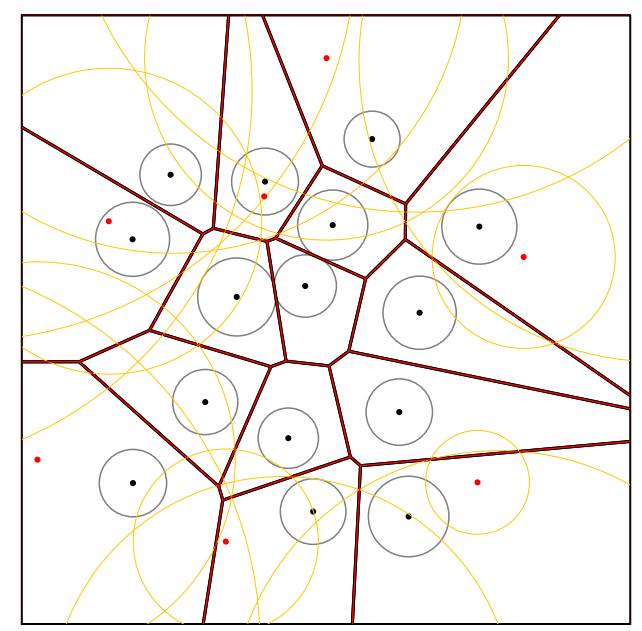

FIGURE 5. Another example of a Laguerre tessellation that is generated by two completely different sets of circles.

As stated above, many applications based on Laguerre tessellations ascribe meaning to the generating circles. For this reason, it is important that we have a method that chooses a solution that satisfies a number of criteria such as those listed above. Some of these criteria are model dependent. Others, however, are fairly universal. In particular, it is almost always desirable to have generating points lie within the cells they generate and it is almost always desirable to have real-valued radii.

We encode these universal criteria into our algorithm by requiring that the generating circles produced all lie within their cells and all have real-valued radii. We call the set of points that satisfy these two criteria $\mathcal{P}$. We then choose the inputs to Algorithm $1,\left(x_{1}, y_{1}, r_{1}, x_{2}\right)$, so that the resulting set of generators, $\mathbf{P}$, belongs to $\mathcal{P}$.

In addition to requiring that the set of generator points lies in $\mathcal{P}$, we also require that the generators satisfy an additional optimality criterion that is determined by the application. In the following, we choose to solve

$$
\min _{\mathbf{P} \in \mathcal{P}} \max _{r \in \mathbf{P}} r .
$$

That is, we wish to find the generator set $\mathbf{P} \in \mathcal{P}$ for which the maximum radius is minimal. 
However, the method we propose is more general. We could, for example, seek to minimize the difference between the average circle area and the average cell area, or minimize the average distance of a generating point from the center of mass of its cell.

Problem (8) is an optimization problem with complicated constraints and many local optima. As a result, it is a difficult problem to solve numerically. In particular, numerical solutions based on local search are very dependent on the choice of initial conditions. Small changes in initial conditions can result in very different generator sets.

Stochastic algorithms tend to outperform deterministic approaches in such settings. In particular, these algorithms are able to escape many local optima and are not so sensitive to initial conditions. We use a stochastic global optimization technique, the cross-entropy (CE) method, which is both effective and straightforward to implement.

\section{CE METHOD FOR INVERTING LA- GUERRE TESSELLATIONS}

The CE method has been successfully applied to many complicated integer non-linear programming and continuous multi-extremal optimization (see, for example, [20]). In this section we describe how the method can be used to determine pertinent (meaningful) Laguerre generators.

The idea behind the CE method is that the global optimum can be described by a degenerate probability density (that is, a density that ascribes all its mass to a single point). The algorithm iteratively generates a sequence of probability densities that converge to this degenerate density. For more details on the method we refer to [16] and [21].

For the minimization problem (8), we wish to find a generator set $\mathbf{P}^{*}$ in the collection all feasible sets $\mathcal{P}$, that attains the minimum maximum radius:

$$
\gamma^{*}=\min _{\mathbf{P} \in \mathcal{P}} \max _{r \in \mathbf{P}} r
$$

where $\mathbf{P}$ is generated via Algorithm 1. Since we are interested in the minimizer $\mathbf{P}^{*}$ rather than the minimum $\gamma^{*}$, we can, in view of Remark 3 , reduce the dimension of this constrained minimization problem from four to three, by setting $r_{1}=0$ in the input of Algorithm 1.

The CE algorithm now comprises the following iterative steps:

1. Generate a random sample of starting values $\left(x_{1}, y_{1}, x_{2}\right)$ according to a multi-variate normal distribution.

2. For each of these starting values generate the set of generators via Algorithm 1 and compute the corresponding maximum radii.
3. Update the parameters of the sampling distribution, based on the best performing samples (the so-called elite samples), using cross-entropy minimization. The best performing samples are those with the smallest maximum radii.

The CE method produces a sequence of parameters $\left\{\left(\boldsymbol{\mu}_{t}, \boldsymbol{\sigma}_{t}^{2}\right)\right\}$ for the multivariate normal sampling distribution and a sequence of levels $\left\{\gamma_{t}\right\}$ decreasing to $\gamma^{*}$. To run the CE method, we need to specify the initial sampling parameters, the sample size $N$, a rarity parameter $\varrho$, and a stopping criterion.

At iteration $t$ we generate $N$ independent samples $\left(x_{1}^{(k)}, y_{1}^{(k)}, x_{2}^{(k)}\right), k=1, \ldots, N$ from a three-dimensional normal distribution, where each component is sampled independently. We write $\left(x_{1}^{(k)}, y_{1}^{(k)}, x_{2}^{(k)}\right) \sim_{\text {iid }}$ $\mathrm{N}\left(\boldsymbol{\mu}_{t}, \boldsymbol{\sigma}_{t}^{2}\right)$, where $\boldsymbol{\mu}_{t}$ is the three-dimensional vector of expectations and $\boldsymbol{\sigma}_{t}^{2}$ is the three-dimensional vector of variances. For the normal distribution the CE updating rule in Step 3 above is particularly easy (see [21]): the expectation vector becomes the vector of sample means of the elite samples, and the vector of variances becomes the vector of sample variances of the elite samples.

The detailed steps of the CE method for inverting Laguerre tessellations are as follows.

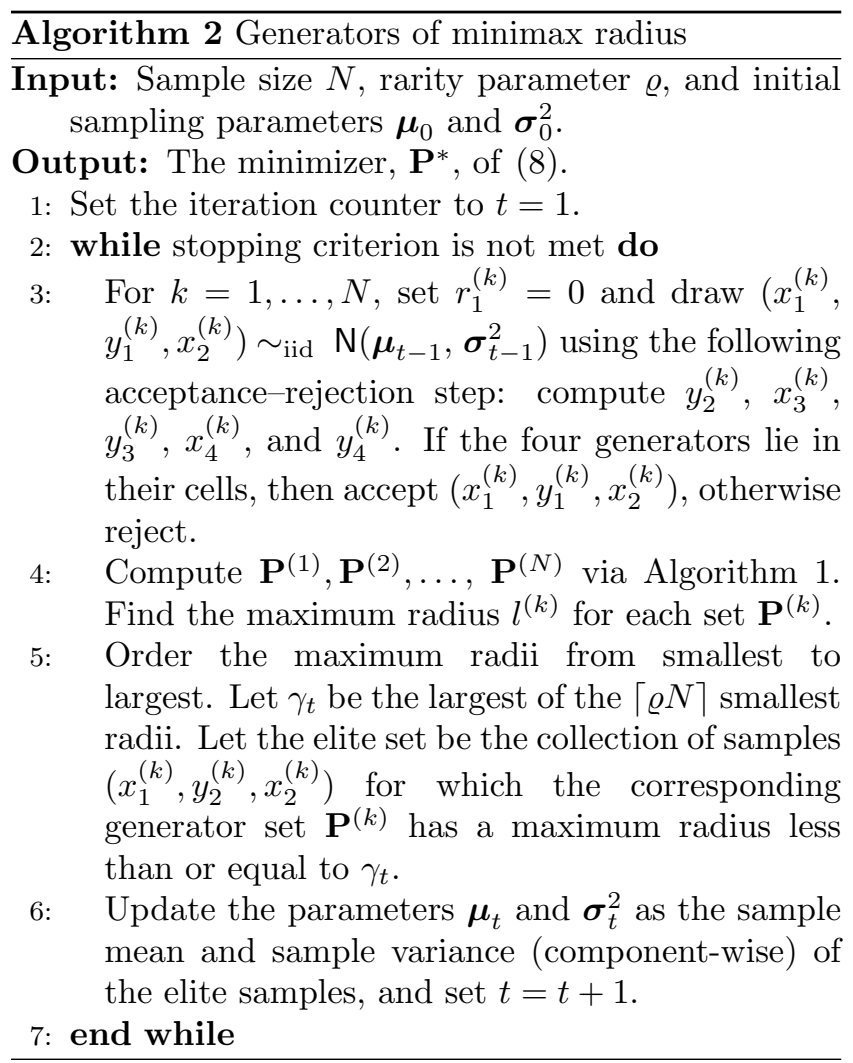

Remark 4. Algorithm 2 uses an acceptancerejection step to constrain the first four generators to lie within their cells. We found experimentally that this is sufficient to ensure that eventually all generators end up within their cells. It is difficult to initially constrain all generators inside their cells, as the search 
TABLE 2. Example 1: Convergence of parameters

\begin{tabular}{cccccc}
$t$ & $\gamma_{t}$ & $\overbrace{}^{c} \boldsymbol{\mu}_{t}$ & $\max \left\{\boldsymbol{\sigma}_{t}\right\}$ \\
\hline 1 & 31.7198 & 93.4043 & 109.6592 & 122.2072 & 4.6120 \\
2 & 21.6147 & 93.0674 & 110.9199 & 123.5051 & 1.3520 \\
3 & 13.5177 & 93.0501 & 111.1407 & 123.7959 & 0.4514 \\
4 & 9.9260 & 93.1264 & 111.1727 & 123.9915 & 0.1348 \\
5 & 8.8108 & 93.1707 & 111.1562 & 124.0233 & 0.0553 \\
6 & 8.5782 & 93.1934 & 111.1419 & 124.0357 & 0.0368 \\
7 & 8.5237 & 93.2111 & 111.1350 & 124.0412 & 0.0221 \\
8 & 8.4993 & 93.2210 & 111.1309 & 124.0441 & 0.0108 \\
9 & 8.4857 & 93.2271 & 111.1286 & 124.0456 & 0.0062 \\
10 & 8.4782 & 93.2301 & 111.1274 & 124.0466 & 0.0034 \\
11 & 8.4742 & 93.2316 & 111.1267 & 124.0469 & 0.0020 \\
12 & 8.4719 & 93.2325 & 111.1263 & 124.0471 & 0.0012 \\
13 & 8.4706 & 93.2331 & 111.1260 & 124.0472 & 0.0007 \\
14 & 8.4699 & 93.2334 & 111.1259 & 124.0473 & 0.0004 \\
15 & 8.4694 & 93.2335 & 111.1258 & 124.0473 & 0.0002 \\
16 & 8.4692 & 93.2336 & 111.1257 & 124.0473 & 0.0001 \\
17 & 8.4690 & 93.2336 & 111.1257 & 124.0473 & 0.0001
\end{tabular}

space will become very small. As far as we are aware, it remains an open theoretical problem whether every Laguerre tessellation admits a solution in which every cell contains one generator point.

A possible stopping criterion (which we have used in Section 5 is to stop when the maximum standard deviation, $\max \left(\boldsymbol{\sigma}_{t}\right)$, is less than some small tolerance $\varepsilon>0$.

\section{NUMERICAL EXPERIMENTS}

In this section, two numerical examples are given to indicate how Algorithm 2 works for inverting Laguerre tessellations. The tessellation data is of the format in Table 1 and can be downloaded from http://www . maths.uq.edu.au/ kroese/Laguerre.

\section{Example 1}

The first example is the running example, whose original generators are shown in Figure 1. In Figures 3 and 5, we saw examples of poorly behaved generators, obtained via Algorithm 1. We now employ Algorithm 2 to obtain a good generator set, whose maximum radius is as small as possible.

In this example, we use cells 2 and 6 as our starting cells (instead of 1 and 2 ; note that cell 1 is not internal here). We choose $\boldsymbol{\mu}_{0}=\left(\mu_{0}^{x_{1}}, \mu_{0}^{y_{1}}, \mu_{0}^{x_{2}}\right)$ as follows, where $\mu_{0}^{x_{1}}$ is the average of the $x$-coordinates of all vertices of $C_{1}, \mu_{0}^{y_{1}}$ is the average of the $y$-coordinates of all vertices of $C_{1}$, and $\mu_{0}^{x_{2}}$ is the average of the $x$-coordinates of all vertices of $C_{2}$. The three initial variances are set to 100 (standard deviation 10), so that most of samples lie inside the cells $C_{2}$ and $C_{6}$.

Table 2 shows the progress of the CE method, where the sample size $N=4000$, rarity parameter $\varrho=0.05$ and stopping criterion $\operatorname{MAX}(\boldsymbol{\sigma})<\varepsilon=10^{-4}$. In Table 2 , $\gamma_{t}$ is the $\lceil N \varrho\rceil$-th largest radius over all maximum radii of each set in $t$-th iteration. Note that the minimum radius of each set is 0 . Figure 6 indicates the difference between our generators and the original ones. While the generator points are very similar, the radii are significantly smaller for the CE generators. The maximum radius reduces from 9.5034 to 8.4690. Also, the minimum radius for the CE case is (always) 0 .

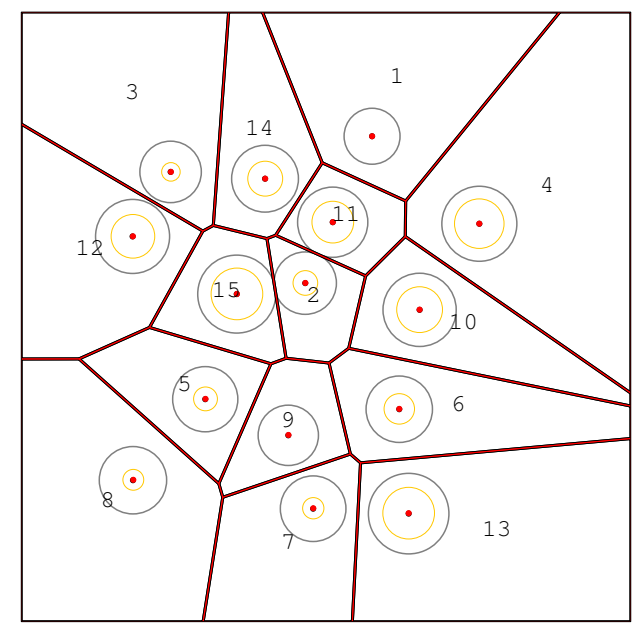

FIGURE 6. Result of Example 1

To show the dependence of the CE algorithm on the parameters, we ran Algorithm 2 with different sets of parameters, such as $N=4000, \varrho=0.1, N=2000, \varrho=$ 0.05 and $N=2000, \varrho=0.1$. The progress of $\gamma_{t}$ as a function of $t$ is shown in Figure 7 . We see a robust behavior with respect to the choice of $\mathrm{CE}$ parameters.

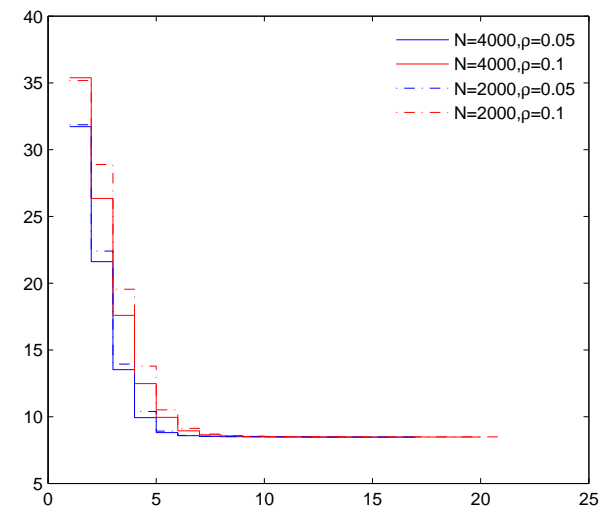

FIGURE 7. CE method for Example 1

\section{Example 2}

In Figure 8, we give a relatively complicated Laguerre tessellation, with 88 cells for which the generators need to be recovered. The original generators are also given in Figure 8. 
TABLE 3. Example 2: Convergence of parameters

\begin{tabular}{cccccc}
$t$ & $\gamma_{t}$ & $\overbrace{592.1691}$ & 131.5233 & $\max \left\{\boldsymbol{\sigma}_{t}\right\}$ \\
\hline 1 & 31.9997 & 142.7428 & 57.16970 \\
2 & 17.7478 & 142.3349 & 57.4951 & 131.2341 & 0.5303 \\
3 & 11.8451 & 142.2002 & 57.5020 & 131.1021 & 0.2092 \\
4 & 9.6158 & 142.1381 & 57.5472 & 131.0550 & 0.0712 \\
5 & 9.1285 & 142.1171 & 57.6062 & 131.0436 & 0.0319 \\
6 & 9.0075 & 142.1075 & 57.6289 & 131.0378 & 0.0149 \\
7 & 8.9754 & 142.1051 & 57.6437 & 131.0365 & 0.0065 \\
8 & 8.9624 & 142.1044 & 57.6500 & 131.0362 & 0.0031 \\
9 & 8.9568 & 142.1041 & 57.6535 & 131.0362 & 0.0015 \\
10 & 8.9539 & 142.1041 & 57.6553 & 131.0363 & 0.0008
\end{tabular}

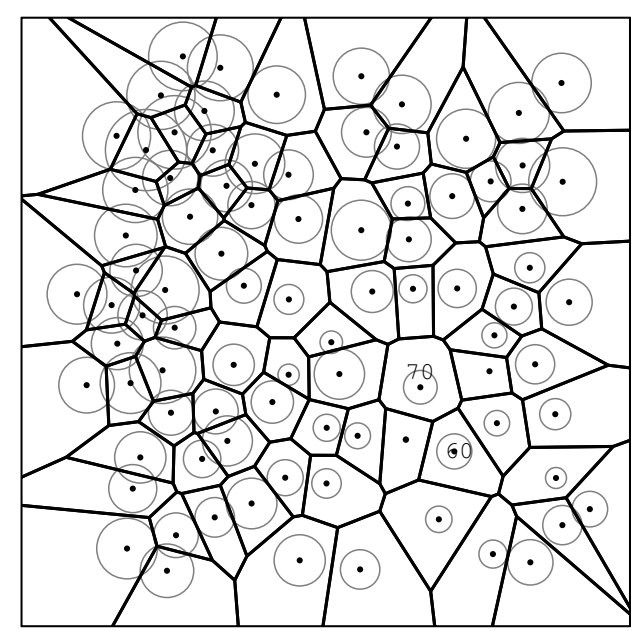

FIGURE 8. Laguerre tessellation for 88 generators

We use cells 60 and 70 as starting cells and set the initial distribution parameters $\boldsymbol{\mu}_{0}$ and $\boldsymbol{\sigma}_{0}$ in the same way as Example 1. As for the parameters of the CE method, we set sample size $N=5000$, rarity parameter $\varrho=0.01$ and stopping criterion $\max (\boldsymbol{\sigma})<$ $\varepsilon=10^{-3}$. The progress of the algorithm is shown in Table 3. Figure 9 shows the behavior of the method under different sets of CE parameters. Even though the processes are a little different, the results are almost the same.

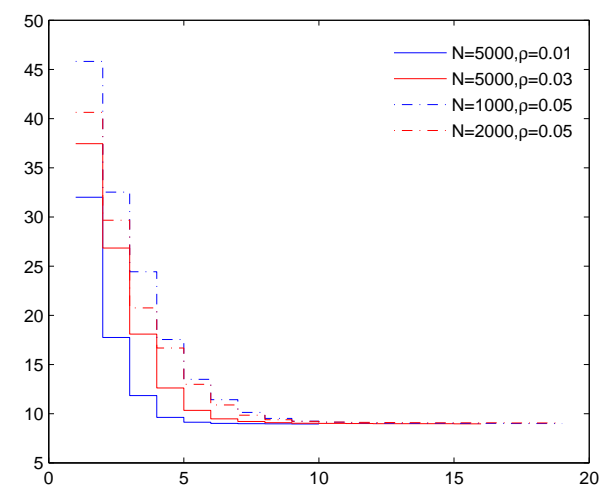

FIGURE 9. CE method for Example 2
The original and CE generators are given in Figure 10, showing very good agreement in location, with again a slightly smaller radii for the $\mathrm{CE}$ case.

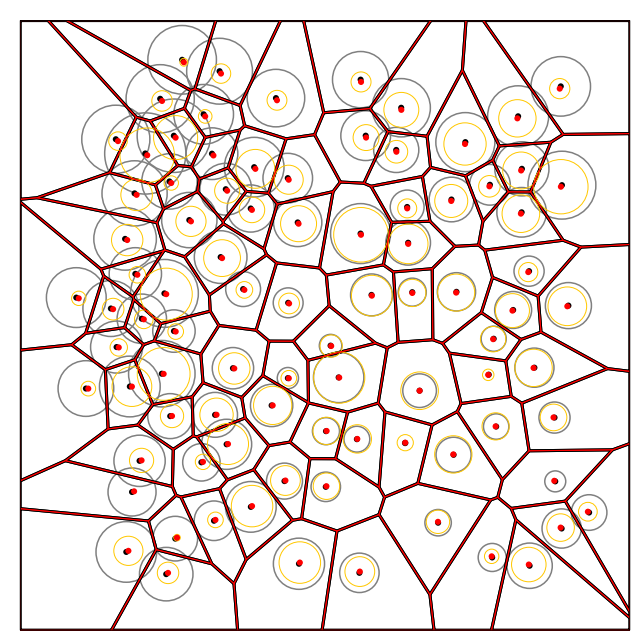

FIGURE 10. Result of Example 2

REMARK 5. The Voronoi tessellation is a special case of the Laguerre tessellation. In the Voronoi case, all the generator weights are equal (and can be assumed to be 0 ). Algorithm 2 is easily adapted to invert $2 \mathrm{D}$ Voronoi tessellations.

As in Algorithm 2, we start from two initial cells, $C_{1}$ and $C_{2}$. We generate sample points in $C_{1}$ and find the corresponding points in $C_{2}$ using the perpendicular bisector. We set the radii of the generating circles in $C_{1}$ and $C_{2}$ to be 0 . We then use the CE method to find points such that the maximum radius of a generating circle is zero. Note, however, that [12] gives a more efficient method for inverting Voronoi tessellations.

\section{CONCLUSIONS AND PROPOSED EX- TENSIONS}

In this paper, we have provided a general method for inverting normal Laguerre tessellations. We have demonstrated that there are often a number of different solutions to this inversion problem. Many of these solutions have limited explanatory power. We proposed a method, based on randomized optimization, for finding pertinent solutions. That is, solutions that satisfy certain criteria imposed by the application. We have given a number of numerical results that demonstrate the effectiveness of this approach.

There are many possible extensions to our work. Our method can be extended to higher dimension. Our method can be applied in a number of fields that use Laguerre tessellations as models, for example materials science and biology. We also believe that the cross-entropy method could also be applied to other tessellations, including those with non-convex cells. 


\section{ACKNOWLEDGEMENTS}

This work was supported by the Australian Research Council under grant number DP0985177.

\section{REFERENCES}

[1] Okabe, A., Boots, B., Sugihara, K., and Chiu, S. N. (2000) Spatial tessellations: concepts and applications of Voronoi diagrams, 2nd edn. edition. J. Wiley \& Sons, Chichester.

[2] Aurenhammer, F. (1987) Power diagrams: properties, algorithms and applications. SIAM Journal on Computing, 16, 78-96.

[3] Lautensack, C. (2007) Random Laguerre Tessellations. $\mathrm{PhD}$ thesis Karlsruhe Institue of Technology.

[4] Tanuma, T., Imai, H., and Moriyama, S. (2001) Revisiting hyperbolic Voronoi diagrams in two and higher dimensions from theoretical, applied and generalized viewpoints. Transactions on Computational Science XIV: Special Issue on Voronoi Diagrams and Delaunay Triangulation, pp. 1-30. Springer Verlag, New York.

[5] Telley, H., Liebling, T. M., and Mocellin, A. (1996) The Laguerre model of grain growth in two dimensions I. Cellular structures viewed as dynamical Laguerre tessellations. Philosophical Magazine B, 73, 395-408.

[6] Telley, H., Libeling, T. M., and Mocellin, A. (1996) The Laguerre model of grain growth in two dimensions II. Examples of coarsening simulations. Philosophical Magazine B, 73, 409-427.

[7] Ogawa, H., Wakai, F., and Waseda, Y. (1996) Molecular dynamics simulation of the model grain boundary structure of polycrystalline materials. Molecular Simulation, 18, 179-192.

[8] Ash, P. F. and Bolker, E. D. (1985) Recognizing Dirichlet tessellations. Geometriae Dedicata, 19, 175206.

[9] Evans, D. G. and Jones, S. M. (1987) Detecting Voronoi (area-of-influence) polygons. Mathematical geology, 19, 523-537.

[10] Aurenhammer, F. (1987) Recognising polytopical cell complexes and constructing projection polyhedra. Journal of Symbolic Computation, 3, 249-255.

[11] Hartvigsen, D. (1992) Recognizing Voronoi diagrams with linear programming. ORSA Journal on Computing, 4, 369-374.

[12] Schoenberg, F. P., Ferguson, T., and Li, C. (2003) Inverting Dirichlet tessellations. The Computer Journal, 46, 76-83.

[13] Adamatzky, A. (1993) Massively parallel algorithm for inverting Voronoi diagram. Neural Netw. World, 5, 385-92.

[14] Lyckegaard, A., Lauridsen, E. M., Ludwig, W., Fonda, R. W., and Poulsen, H. F. (2011) On the use of Laguerre tessellations for representations of $3 \mathrm{D}$ grain structures. Advanced Engineering Materials, 13, 165170.

[15] Spettl, A., Werz, T., Krill, C. E., and Schmidt, V. (2013) Parametric representation of 3D grain ensembles in polycrystalline microstructures. Submitted.

[16] Rubinstein, R. Y. and Kroese, D. P. (2004) The CrossEntropy method: A unified approach to combinatorial optimization, Monte-Carlo simulation and machine learning. Springer, New York.

[17] Gervois, A., Oger, L., Richard, P., and Troadec, J. P. (2002) Voronoi and radical tessellations of packings of spheres. Computational Science - ICCS 2002, pp. 95104. Springer, Berlin.

[18] Sadoc, J., Jullien, R., and Rivier, N. (2003) The laguerre polyhedral decomposition: application to protein folds. The European Physical Journal BCondensed Matter and Complex Systems, 33, 355-363.

[19] Aurenhammer, F. (1987) A criterion for the affine equivalence of cell complexes in $\mathbb{R}^{d}$ and convex polyhedra in $\mathbb{R}^{d+1}$. Discrete and Computational Geometry, 2, 49-64.

[20] Botev, Z. I., Kroese, D. P., Rubinstein, R. Y., and L'Ecuyer, P. (2013) The Cross-Entropy method for optimization. Machine Learning: Theory and Applications, V. Govindaraju and C.R. Rao, Eds, Chennai: Elsevier B.V., 31, 35-59.

[21] Kroese, D. P., Porotsky, S., and Rubinstein, R. Y. (2006) The Cross-Entropy method for continuous multi-extremal optimization. Methodology and Computing in Applied Probability, 8, 383-407. 